\title{
Automotive landscape 2030+ - Pole position for Germany in autonomous driving
}

Steffen Gänzle, A.T. Kearney GmbH

This manuscript is not available according to publishing restriction.

Thank you for your understanding. 